\title{
Euler-Bernoulli Nanobeam Welded to a Compressible Semi-Infinite Substrate
}

\author{
Pietro Di Maida ${ }^{1}$ and Federico Oyedeji Falope ${ }^{2}$ \\ ${ }^{1}$ Department of Sciences and Methods for Engineering (DISMI), University of Modena and Reggio Emilia, \\ Via Amendola 2, 42122 Reggio Emilia, Italy \\ ${ }^{2}$ Department of Engineering Enzo Ferrari (DIEF), University of Modena and Reggio Emilia, Via P. Vivarelli 10, Int 27, \\ 41125 Modena, Italy
}

Correspondence should be addressed to Pietro Di Maida; pietro.dimaida@unimore.it

Received 23 December 2015; Accepted 5 October 2016

Academic Editor: Julius Kaplunov

Copyright (C) 2016 P. Di Maida and F. O. Falope. This is an open access article distributed under the Creative Commons Attribution License, which permits unrestricted use, distribution, and reproduction in any medium, provided the original work is properly cited.

\begin{abstract}
The contact problem of an Euler-Bernoulli nanobeam of finite length bonded to a homogeneous elastic half plane is studied in the present work. Both the beam and the half plane are assumed to display a linear elastic behaviour under infinitesimal strains. The analysis is performed under plane strain condition. Owing to the bending stiffness of the beam, shear and peeling stresses arise at the interface between the beam and the substrate within the contact region. The investigation allows evaluating the role played by the Poisson ratio of the half plane (and, in turn, its compressibility) on the beam-substrate mechanical interaction. Different symmetric and skew-symmetric loading conditions for the beam are considered, with particular emphasis to concentrated transversal and horizontal forces and couples acting at its edges. It is found that the Poisson ratio of the half plane affects the behaviour of the interfacial stress field, particularly at the beam edges, where the shear and peel stresses are singular.
\end{abstract}

\section{Introduction}

The mechanical interaction between bars, strips, rings, thin films, and so forth and an elastic substrate has been widely investigated because of its great importance in many practical engineering tasks. As an example, in the framework of civil engineering, the load transfer between FRP sheets (or other reinforcing elements like discrete fibres [1,2]) applied to existing concrete structures is often performed by modeling the reinforcing stiffeners as $2 \mathrm{D}$ membranes [3-6] welded to half plane (for the equilibrium configurations of cylindrical and cubic bodies [2]). By following such an approach, retrofitted concrete structures [7], elastic foundations [8-10], rigid road pavements [11], and retrofitted masonry elements [12,13] have been studied (for the behaviour of high performance concrete based on fly ash, see [14]).

In microelectronics, various NEMS and MEMS involve thin films and coatings [5, 15-17] (examples about numerical studies performed on thin films can be found in $[6,18]$ ), and the stress concentrations arising in the neighbourhood of geometric discontinuities and/or across bimaterial interfaces provide useful information about the risk of occurrence of fracture phenomena $[19,20]$ in such devices and, in turn, about their stability in time [21, 22].

In mechanical engineering, the film-substrate contact mechanics is relevant in order to study the mechanical response of thermal barriers and protective alloys typically involved in a variety of mechanical devices, like blades of centrifugal pump impellers, components of compressors, gas turbines, and so forth. In most of these examples, the problem can be studied by neglecting the bending stiffness of the covering; namely, the coating is modeled like a membrane element. However, in a wide class of mechanical systems, the flexural behaviour of the covers can not be neglected without introducing rough approximations. As an example, the flexural behaviour must be taken into account to properly study beams [23] and multilayer systems [24]. As an example, foundations of buildings are often characterized by high values of flexural rigidity and, typically, they are simulated as beams or plates resting on an elastic support $[8,9]$. 
Similarly, the mechanical behaviour of MEMS or NEMS based on coatings characterized by small length-to-thickness ratio must be studied by taking into account the bending stiffness of the layers.

The contact problem of an Euler-Bernoulli beam perfectly bonded to an elastic half plane has been performed in [23]. These authors carried out their investigation by considering an incompressible half plane (i.e., by considering $v_{s}=$ 0.5 , where $\nu_{s}$ is the Poisson ratio of the substrate), thus finding a nonoscillatory behaviour of the interfacial stress field. This allowed the authors to straightforwardly express the unknown peel and shear stresses as infinite series of Chebyshev polynomials having square-root singularities at the edges of the beam.

Studying the singular nature of the stress and strain fields in such contact problems is an important topic $[6,25]$ in order to assess if such systems are resistant or prone to delamination, interfacial crack propagation, and other damage phenomena [5, 26-28].

In the present work, the effect induced by the Poisson ratio of the half plane on the beam-half plane mechanical interaction has been studied. The oscillatory index characterizing the interfacial stress field is found to depend on the Poisson ratio of the half plane only. The strain compatibility condition leads to a system of two integral equations, which is reduced to algebraic systems by expanding the interfacial stresses in series of orthogonal Jacobi polynomials having complex index. In the present investigation, both the beam and the half plane have been assumed homogeneous bodies displaying linear elastic behaviour. However, the analysis can be extended to nonhomogeneous bodies by following the approach performed in [29-31]. The time dependence can be taken into account also (for a general approach concerning the nonlocal behaviour of functionally graded materials see, e.g., $[32,33])$.

The paper is organized as follows. The formulation of the problem is given in Section 2. The main results are presented and discussed in Section 3. Finally, conclusions are reported in Section 4.

\section{Formulation of the Problem}

The problem of Euler-Bernoulli nanobeams bonded to an isotropic elastic half plane is formulated in the present section. The reference system is centred at the middle span of the beam, as shown in Figure 1. Perfect adhesion between the half plane and beam is assumed. Both the axial and flexural stiffness of the beam are taken into account. The beam and the underlying half plane have an isotropic constitutive law, characterized by the following elastic constants $E_{s}, E_{b}, v_{s}$, and $v_{b}$ denoting the Young moduli and the Poisson ratio, respectively (the subscript $i$, with $i=b, s$ stands for beam or semi-infinite substrate, resp.). Furthermore, $h$, and $2 a$ denote the height and the total length of the beam, respectively, whereas $A$ and $J$ indicate the area and the moment of inertia of the beam cross section.

The shear and peeling stresses, $\tau(y)$ and $q(y)$, respectively (Figure 2), which arise along the contact region, represent the unknown of the problem. In the following, the apex prime $\left(^{\prime}\right)$

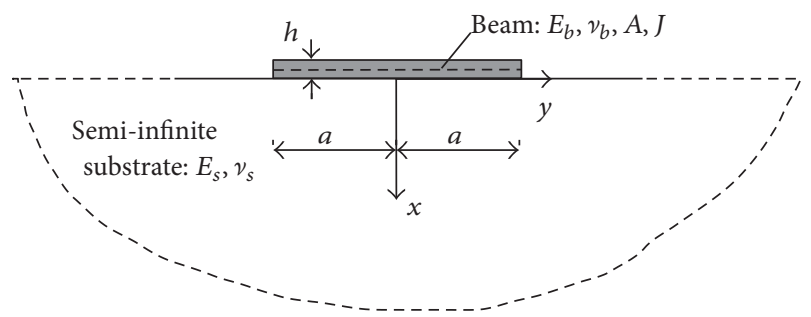

FIGURE 1: Semi-infinite substrate and beam geometry.

denotes the derivative with respect to the spatial coordinate $y$.

By considering the symmetric as well as skew-symmetric loading cases reported in Figure 2, the balance condition of the beam along $y \in[-a,+a]$ leads to

$$
\begin{aligned}
N(y)= & N^{*}+\int_{y}^{a} \tau(t) d t \\
T(y)= & -T^{*}+\int_{y}^{a} q(t) d t \\
M(y)= & M^{*}+T^{*}(a-x)+\frac{h}{2} \int_{y}^{a} \tau(t) d t \\
& +\int_{y}^{a} q(t)(y-t) d t,
\end{aligned}
$$

where $N, T$, and $M$ are the internal axial force, shear force, and bending moment of the beam, respectively, whereas $N^{*}$, $T^{*}$, and $M^{*}$ are the corresponding external concentrated loads acting at the beam edges. By using the equilibrium conditions (1) and the isotropic elastic constitutive law, the axial strain $\varepsilon_{y}^{b}$, and the slope $\varphi^{b}$, take the expressions

$$
\begin{aligned}
& \varepsilon_{y}^{b}(y)=\frac{N^{*}}{E_{b} A}+\frac{1}{E_{b} h} \int_{y}^{a} \tau(t) d t+\frac{h}{2 E_{b} J}\left\{M^{*}\right. \\
&+\left.T^{*}(a-y)+\int_{y}^{a}\left[\frac{h}{2} \tau(t)+q(t)(y-t)\right]\right\} d t, \\
& \varphi^{b}(y)=\frac{1}{E_{b} J_{b}}\left\{y M^{*}+T^{*}\left(y-\frac{y^{2}}{2}\right)\right. \\
&+\frac{h}{2}\left[y \int_{y}^{a} \tau(t) d t+\int_{0}^{y} t \tau(t) d t\right] \\
&\left.-\frac{1}{2}\left[\int_{0}^{a} t^{2} q(t) d t-\int_{y}^{a}(t-y)^{2} q(t) d t\right]\right\}+\tilde{\varphi},
\end{aligned}
$$

where $\widetilde{\varphi}$ is an unknown integration constant. Concerning the semi-infinite substrate, the longitudinal strain and slope at the contact region are known in closed form [34] as

$$
\begin{aligned}
\varepsilon_{y}^{s}(y)= & \frac{2\left(1-v_{s}^{2}\right)}{E_{s} \pi} \int_{-a}^{+a} \frac{\tau(t)}{y-t} d t \\
& +\frac{\left(1-2 v_{s}\right)\left(1+v_{s}\right)}{E_{s}} q(y),
\end{aligned}
$$




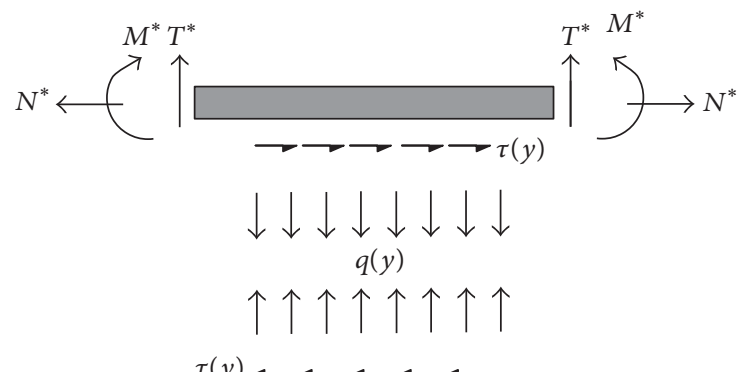

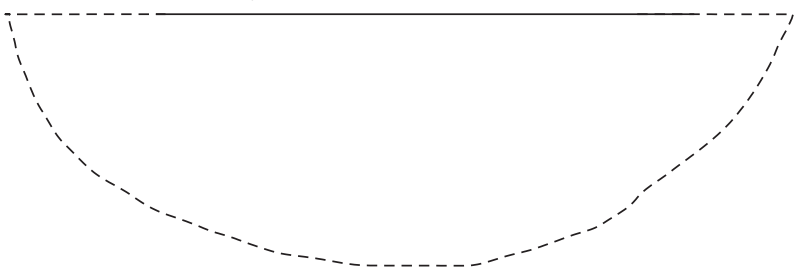

(a) Symmetric
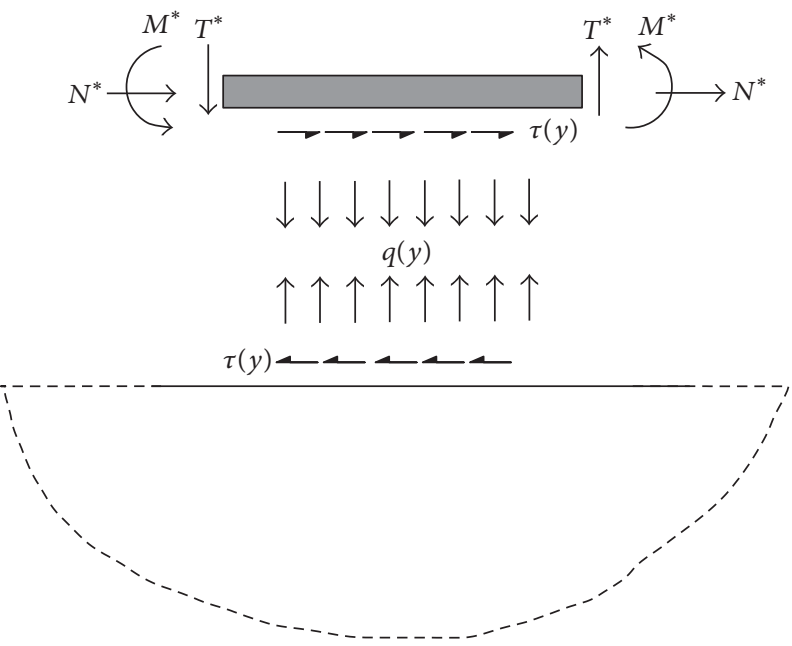

(b) Skew-symmetric

FIgURE 2: Mechanical interaction between the beam and the substrate for symmetric and skew-symmetric loading cases.

$$
\begin{aligned}
\varphi^{s}(y)= & -\frac{\left(1-2 v_{s}\right)\left(1+v_{s}\right)}{E_{s}} \tau(y) \\
& +\frac{\left(1-v_{s}^{2}\right)}{E_{s} \pi} \int_{-a}^{+a} \frac{q(t)}{y-t} d t .
\end{aligned}
$$

Thus, the unknowns of the problem are the stress distributions $\tau(y)$ and $q(y)$ at the interface within the contact region.

The interfacial shear and peeling stresses can be straightforwardly expanded in series of Jacobi orthogonal polynomials,

$$
\begin{aligned}
& \tau(y)=E_{s}(a+y)^{p}(a-y)^{p} \sum_{n=0}^{\infty} C_{n} P_{n}^{(p, p)}\left(\frac{y}{a}\right), \\
& q(y)=E_{s}(a+y)^{p}(a-y)^{p} \sum_{n=0}^{\infty} D_{n} P_{n}^{(p, p)}\left(\frac{y}{a}\right),
\end{aligned}
$$

where $P_{n}^{(p, p)}(y / a)$ represents the Jacobi polynomial of order $n$ (see Appendix) and $p$ is the order of the singularity of the stress field at the beam edges, namely, at $y= \pm a$. Indeed, as shown by [23],

$$
p=-\frac{1}{2}+\frac{i \epsilon}{2 \pi}
$$

with $\epsilon=\log \left(3-4 v_{s}\right)$ or $\epsilon=\log \left(3-v_{s}\right) /\left(1+v_{s}\right)$ for a plane strain or plane stress state, respectively.

The problem is governed by the following compatibility equations between the beam and the substrate:

$$
\begin{gathered}
\varepsilon_{y}^{b}(y)=\varepsilon_{y}^{s}(y), \\
\varphi_{y}^{b}(y)=\varphi_{y}^{s}(y) .
\end{gathered}
$$

By introducing (2)-(4) into (6) and truncating series (4) to the first $N$ elements, the imposition of the compatibility conditions at $N+1$ collocation points $y_{k}$ over the contact region allows reducing the system of singular integral equations (6) into a linear algebraic system for the unknown $C_{n}$ and $D_{n}$. The collocation points $y_{k}$, with $k=1,2, \ldots, N+1$, have been set as the roots of the Chebyshev polynomial of first kind of order $N+1$,

$$
y_{k}=\cos \left[\frac{\pi k}{2(N+1)}\right] \text {. }
$$

Once the coefficients $C_{n}$ and $D_{n}$ are found, the strain and displacement fields can be determined and, in turn, the internal forces in the beam can be assessed. The displacement components along the substrate surface [34] take the form

$$
\begin{aligned}
u^{s}(y)= & \frac{\left(1-2 v_{s}\right)\left(1+v_{s}\right)}{2 E_{s}}\left[\int_{-a}^{y} q(t) d t-\int_{y}^{+a} q(t) d t\right] \\
& -\frac{2\left(1-v_{s}^{2}\right)}{\pi E_{s}} \int_{-a}^{a} \tau(t) \ln |y-t| d t \\
v^{s}(y)= & \frac{\left(1-2 v_{s}\right)\left(1+v_{s}\right)}{2 E_{s}}\left[\int_{-a}^{y} \tau(t) d t-\int_{y}^{+a} \tau(t) d t\right] \\
& -\frac{2\left(1-v_{s}^{2}\right)}{\pi E_{s}} \int_{-a}^{a} q(t) \ln |y-t| d t,
\end{aligned}
$$

where $u$ and $v$ represent the longitudinal and transversal displacement. The displacement field of the beam reads

$$
\begin{gathered}
u^{b}(y)=\tilde{u}+\frac{N^{*}}{E_{b} A} y+\left(\frac{1}{E_{b} A}+\frac{h^{2}}{4 E_{b} J}\right)\left[y \int_{y}^{a} \tau(t) d t\right. \\
\left.+\int_{0}^{y} t \tau(t) d t\right]+\frac{h}{4 E_{b} J}\left[2 M^{*} y+T^{*}\left(2 a y-y^{2}\right)\right. \\
\left.+\int_{y}^{a} q(t)(y-t)^{2} d t-\int_{0}^{a} t^{2} q(t) d t\right],
\end{gathered}
$$




$$
\begin{aligned}
& v^{b}(y)=\frac{1}{2 E_{b} J}\left\{-M^{*} y^{2}-\frac{T^{*}}{3}\left(3 a y^{2}-y^{3}\right)\right. \\
& -h\left[\int_{0}^{y} t(y-t) \tau(t) d t\right] \\
& -h\left[\frac{y^{2}}{2} \int_{y}^{a} \tau(t) d t+\frac{1}{2} \int_{0}^{y} t^{2} \tau(t) d t\right] \\
& +y \int_{0}^{a} t^{2} q(t) d t-\int_{y}^{a} q(t)\left(y t^{2}+\frac{y^{3}}{3}-y^{2} t\right) d t \\
& \left.-\int_{0}^{y} \frac{q(t)}{3} d t\right\}+\widetilde{v}-\tilde{\varphi} y .
\end{aligned}
$$

All the integrals involved in the above expressions can be evaluated in closed form (see Appendix).

The strength of the interfacial stresses at the beam ends can be assessed through the peeling and shear stress intensity factors $K_{I}$ and $K_{I I}$ :

$$
\begin{aligned}
K_{I}( \pm a) & =\lim _{y \rightarrow \pm a} \frac{q(y)}{(a \mp y)^{p}} \\
& = \pm 2^{p} E_{s} a^{p} \sum_{n=0,2, \ldots,}^{N} D_{n} P_{n}^{(p, p)}( \pm 1), \\
K_{I I}( \pm a) & =\lim _{y \rightarrow \pm a} \frac{\tau(y)}{(a \mp y)^{p}} \\
& = \pm 2^{p} E_{s} a^{p} \sum_{n=1,3, \ldots,}^{N} C_{n} P_{n}^{(p, p)}( \pm 1) .
\end{aligned}
$$

\section{Results and Discussion}

The behaviour of the beam-substrate system under the symmetric and skew-symmetric loading conditions reported in Figures 2(a) and 2(b) has been investigated. The series expansions (4) have been truncated to 13 terms and the compatibility conditions (6) have been imposed at 14 collocation points within the contact region. Refereed to the dimensionless spatial coordinate $\xi=y / a$, Figures $3-5$ show the dimensionless shear and peeling stress distributions due to different symmetric or skew-symmetric loading cases.

For the beam loaded with two axial forces acting at the film edges, the shear stress (Figure 3) shows a monotonic trend over the entire contact region. Conversely, the behaviour displayed by the peeling tractions is not monotonic as they change sign in the neighbourhood of the film edge.

For the case of shear forces acting at the beam edges, the shear contact stresses are reported in Figure 4. As shown, the results are almost invariant with respect to the Poisson ratio of the substrate, with particular reference to the peeling stress. The interfacial shear stress displays a nonmonotonic trend, in particular for the loading case of two skew-symmetric shear forces, except for $v_{s}=0.5$, namely, for an incompressible half plane, as found by [23]. The loading case of two symmetric couples acting at the beam edges induce an almost linear trend for the shear stress (Figure 5) in the region closer to the middle of the beam for any value assumed by the Poisson ratio of the half plane. Conversely, when two skew-symmetric couples act at the beam ends, the shear stress decreases as the Poisson ratio increases. Also for the loading cases of two end couples, the Poisson ratio affects the shear stress more than the peel tractions.

The trend exhibited by the peeling distribution on the contact surface allows satisfying the balance of momentum induced by shear stress along contact region and the external load acting on the beam.

\section{Conclusions}

The contact problem of an Euler-Bernoulli nanobeam perfectly welded to a homogeneous elastic half plane has been addressed in the present work. The problem has been solved by expressing the unknown shear and peel interfacial stresses as infinite series of Jacobi orthogonal polynomials. The compatibility condition between the strains of the beam and those of the underlying half plane leads to a system of two Fredholm integral equations with Cauchy kernel. The complex singular behaviour of such stresses has been handled by assuming properly the singularity of the stress field at the beam edges. This allows reducing the singular integral equations into a linear system of algebraic equations, which has been solved for the unknown coefficients of the interfacial stress field. The shear and peel stresses have been evaluated for different values of the Poisson ratio for the half plane, thus finding the effect played by this elastic parameter on the beam-substrate mechanical interaction. In particular, it has been shown that the Poisson ratio of the half plane affects mostly the shear stress distribution than the behaviour of the peel tractions. For both symmetric or skew-symmetric couples or shear forces acting at the beam edges Poisson ratio does not affect significantly the mechanical response of the system.

The analysis allows properly assessing the stress concentrations at the edges of the beam (note that high stress levels can increase the time-dependent effects, as shown in [35-37] for concrete frames), an important task in many engineering problems involving beams and plates bonded to an elastic support. For example, the obtained results can be used to properly design FRP stiffeners applied to concrete structures that are prone to various damaging phenomena (see, e.g., [38]). It should be noted that the case of a thermal variation $\Delta T$ (thermal variations can sensibly affect the mechanical behaviour of structural elements, as shown in $[39,40]$ ) acting on the beam (for the effect produced by thermal loads on concrete structures, see, e.g., [38]) can be studied as the loading case of two symmetric axial end forces $N$ by assuming

$$
N=\left[\left(1+v_{b}\right) \alpha_{b}-\left(1+v_{s}\right) \alpha_{s}\right] E A \Delta T,
$$

where $\alpha$ denotes the coefficient of thermal expansion. Note also that the contact mechanics of a nonhomogeneous composite beam with random composition and microstructure should be investigated by following the approach reported in $[41,42]$. 


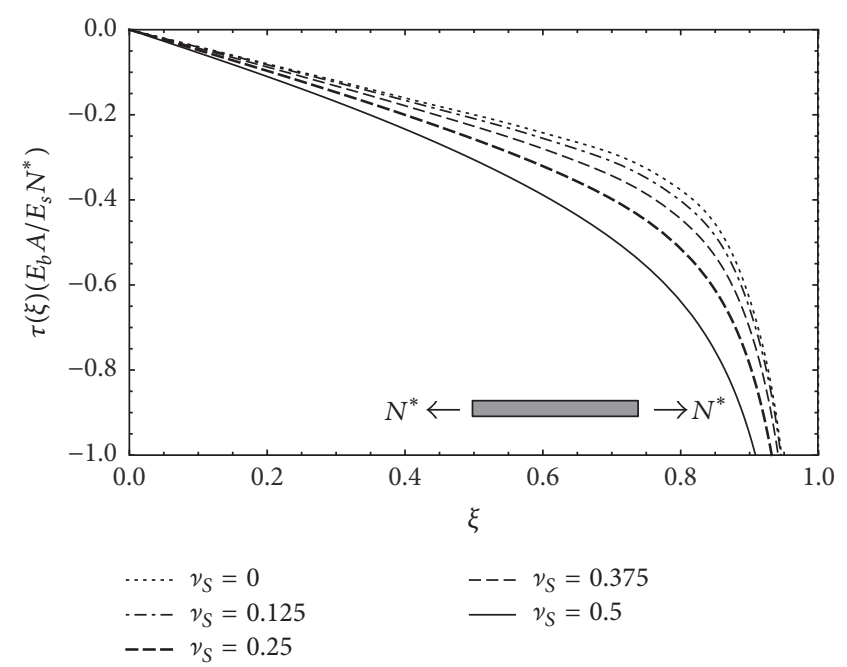

(a) Shear stress for symmetric loading case

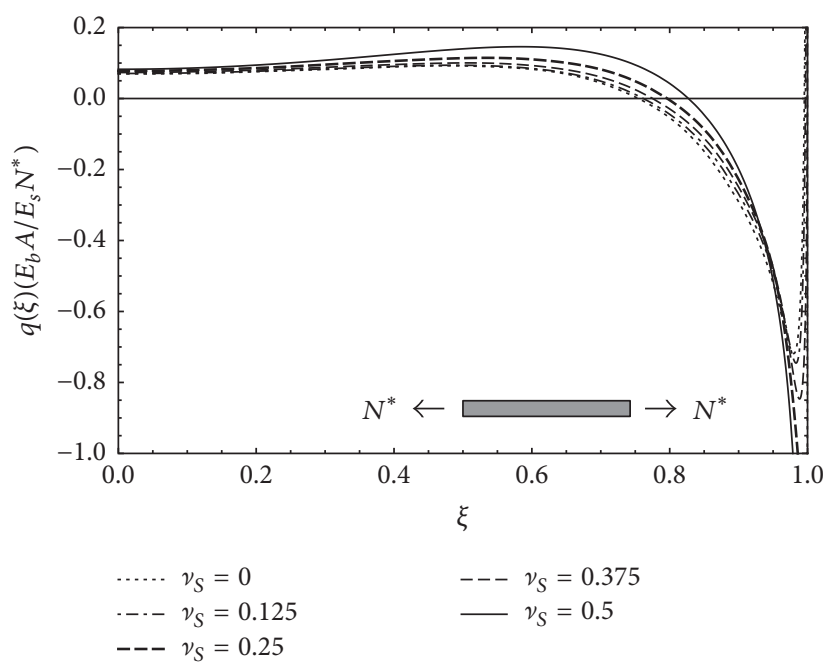

(c) Peeling stress for symmetric loading case

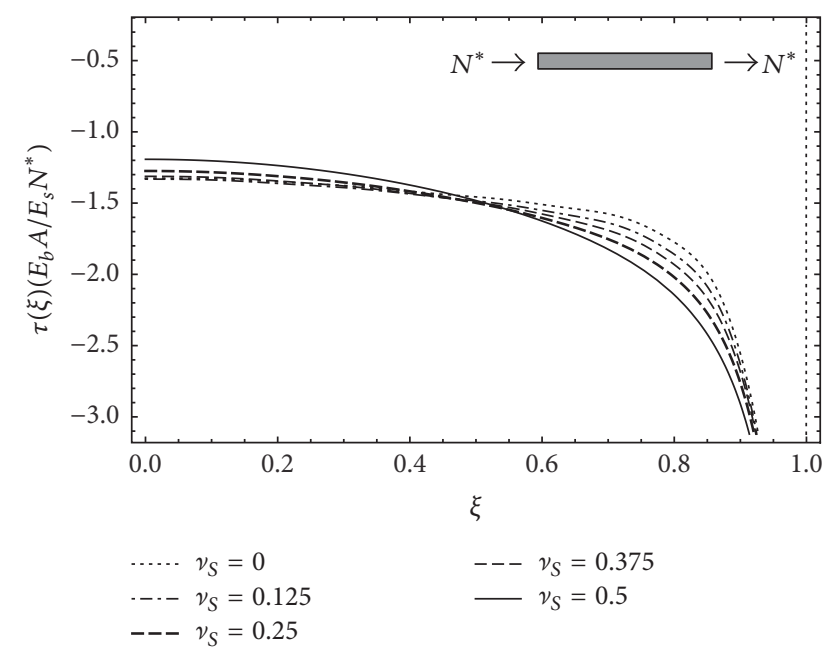

(b) Shear stress for skew-symmetric loading case

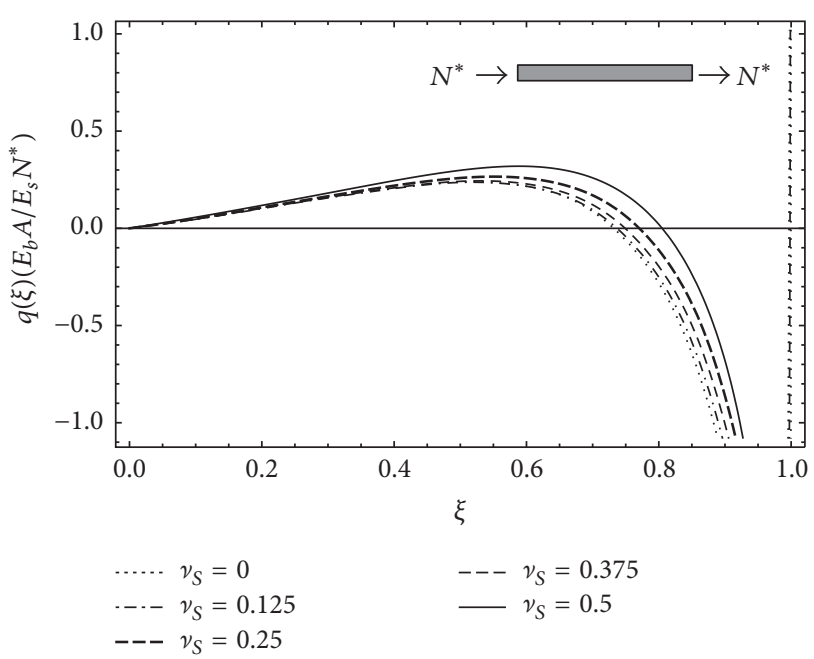

(d) Peeling stress for skew-symmetric loading case

FIGURE 3: Dimensionless mechanical interactions of the beam subjected to axial forces acting at the edges.

\section{Appendix}

\section{Jacobi Polynomials}

The Jacobi polynomial $P_{n}^{(\alpha, \beta)}(t)$ of order $n$ is defined as [43]

$$
\begin{aligned}
& P_{n}^{(\alpha, \beta)}(t)=\frac{\Gamma(\alpha+n+1)}{n ! \Gamma(\alpha+\beta+n+1)} \\
& \cdot \sum_{m=0}^{n}\left(\begin{array}{l}
n \\
m
\end{array}\right) \frac{\Gamma(\alpha+\beta+n+m+1)}{\Gamma(\alpha+m+1)}\left(\frac{t-1}{2}\right)^{m},
\end{aligned}
$$

where $\Gamma$ is the Euler gamma function. In the special case of $\alpha=\beta$ equal to 0 or $1 / 2$ the Jacobi polynomials reduce to the Legendre or Chebyshev polynomials, respectively. Another possible definition of the Jacobi polynomials is provided by Rodrigues' formula,

$$
\begin{aligned}
& P_{n}^{(\alpha, \beta)}(t)=\frac{(-1)^{n}}{2^{n} n !}(1-t)^{-\alpha} \\
& \cdot(1+t)^{-\beta} \frac{d^{n}}{d t^{n}}\left[(1-t)^{\alpha}(1+t)^{\beta}\left(1-t^{2}\right)^{n}\right] .
\end{aligned}
$$

or, equivalently [44],

$$
\begin{aligned}
& P_{n}^{(\alpha, \beta)}(t) \\
& \quad=\frac{(\alpha+1)_{n}}{n !}{ }_{2} F_{1}\left(-n, 1+\alpha+\beta+n ; \alpha+1 ; \frac{1-t}{2}\right),
\end{aligned}
$$

where ${ }_{2} F_{1}$ represents the hypergeometric function for $|t|<$ 1. The orthogonal properties of Jacobi polynomials over $[-1,+1]$ domain provide the following identities used in 


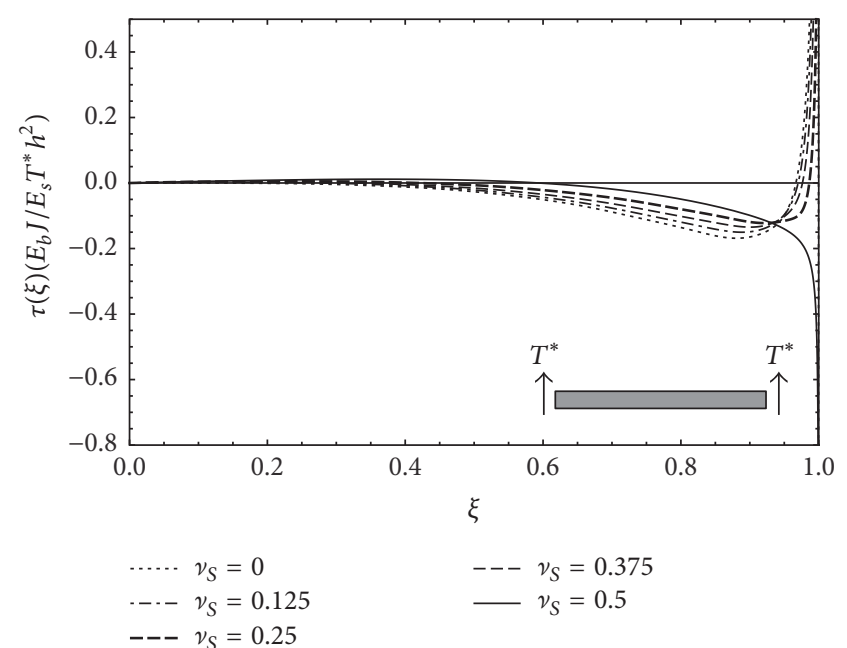

(a) Shear stress for symmetric loading case

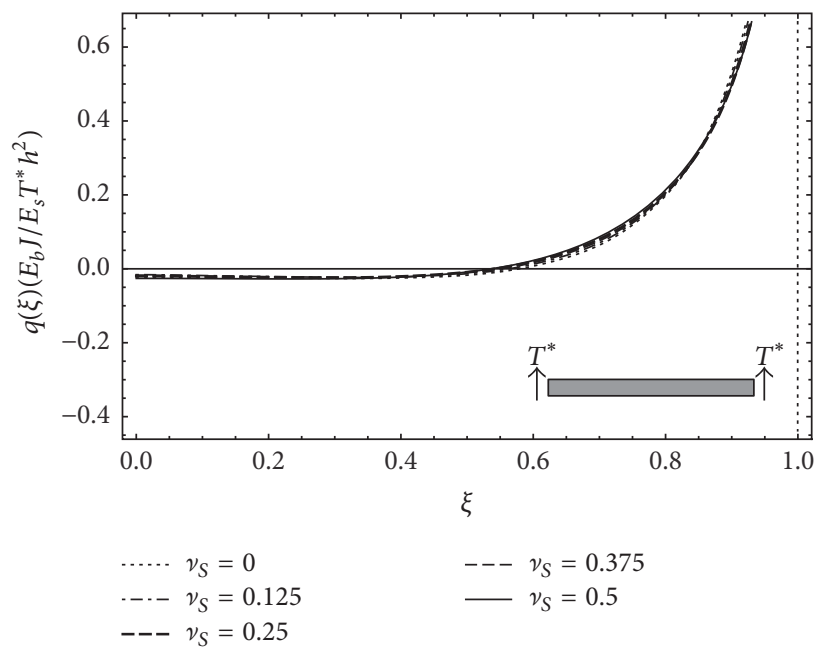

(c) Peeling stress for symmetric loading case

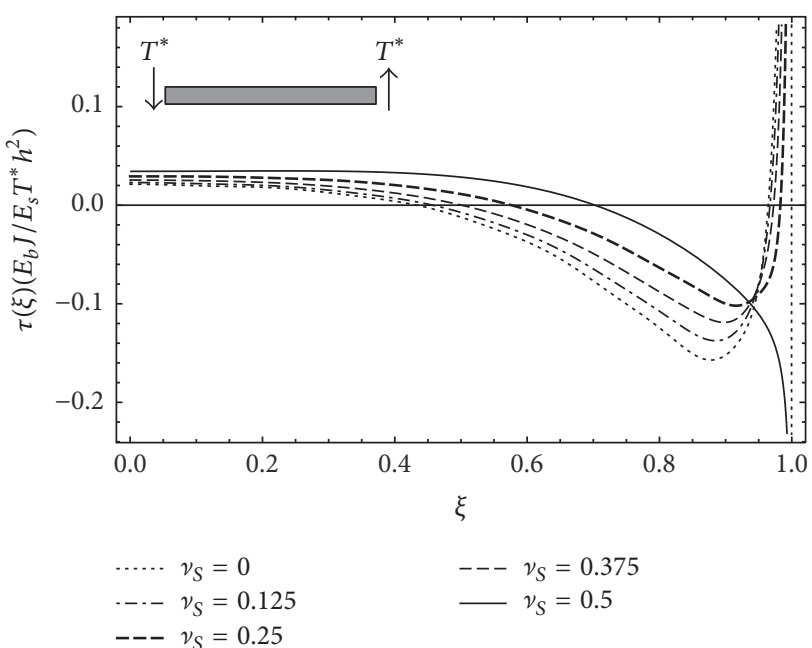

(b) Shear stress for skew-symmetric loading case

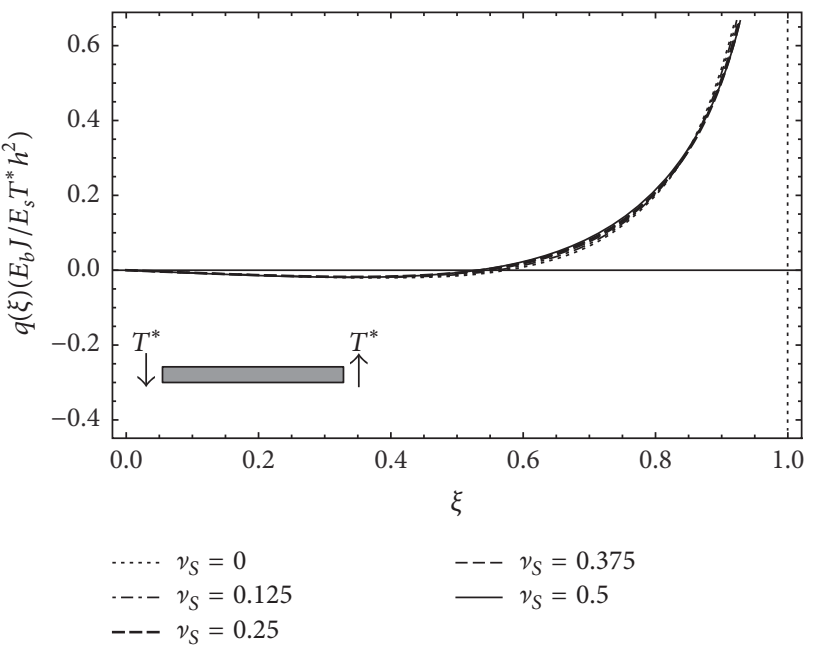

(d) Peeling stress for skew-symmetric loading case

Figure 4: Dimensionless mechanical interactions of the beam subjected to shear forces acting at the edges.

Section 2 to evaluate in closed form the integrals involved in the compatibility equations [45]:

$$
\begin{aligned}
& \int_{-1}^{+1}(1+t)^{p}(1-t)^{p} P_{n}^{(p, p)}(t) P_{m}^{(p, p)}(t) d t \\
& =\frac{2^{2 p+1} \Gamma^{2}(p+n+1) \delta_{n m}}{n !(2 p+1+2 n) \Gamma(2 p+1+n)}, \\
& \int_{-1}^{+1}(1+t)^{p}(1-t)^{p} P_{n}^{(p, p)}(t) d t \\
& =\sqrt{\pi} \frac{\Gamma(p+1)}{\Gamma(p+(3 / 2))} \delta_{n 0}, \\
& \int_{-1}^{+1} t(1+t)^{p}(1-t)^{p} P_{n}^{(p, p)}(t) d t
\end{aligned}
$$

$$
\begin{aligned}
& \quad=\sqrt{\pi} \frac{\Gamma(p+2)}{\Gamma 2(p+(5 / 2))} \delta_{n 0}, \\
& \int_{-1}^{r}(1+t)^{p}(1-t)^{p} P_{n}^{(p, p)}(t) d t \\
& \quad-\int_{r}^{+1}(1+t)^{p}(1-t)^{p} P_{n}^{(p, p)}(t) d t \\
& \quad= \begin{cases}2 t_{2} F_{1}\left(\frac{1}{2},-p, \frac{3}{2}, r^{2}\right), & \text { for } n=0 \\
-\frac{\left(1-r^{2}\right)^{p+1}}{n} P_{n-1}^{(p+1, p+1)}(r), & \text { for } n=1,2, \ldots,\end{cases}
\end{aligned}
$$

where $\delta_{n m}$ represents the Kronecker delta. 


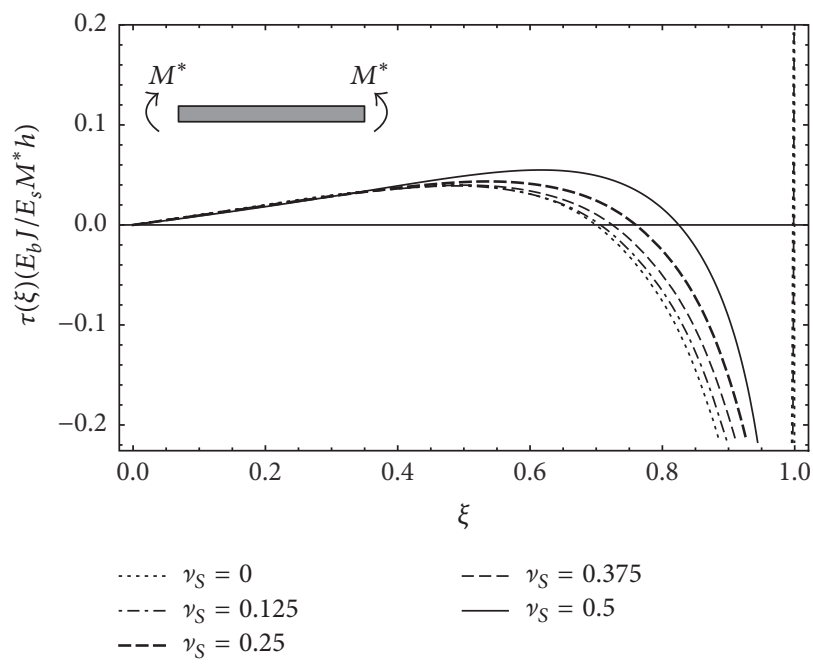

(a) Shear stress for symmetric loading case

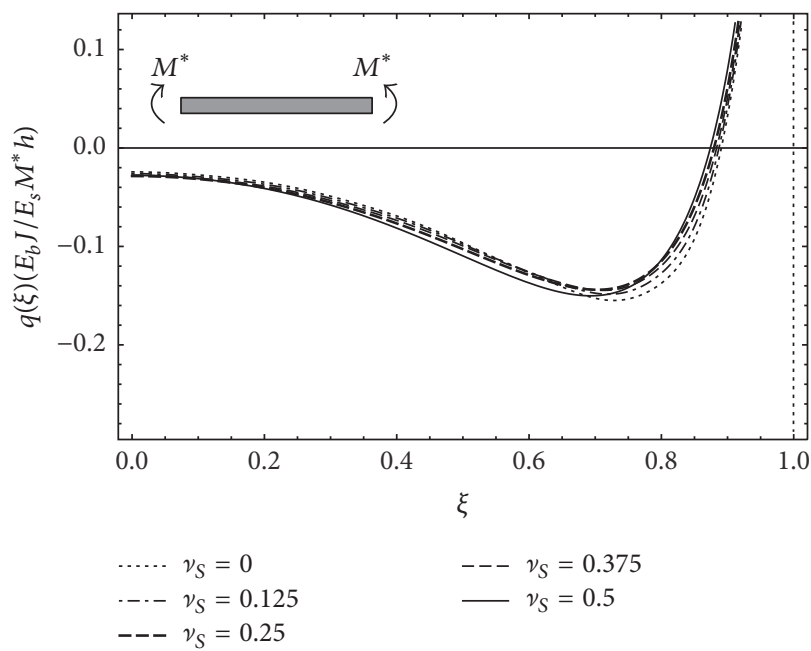

(c) Peeling stress for symmetric loading case

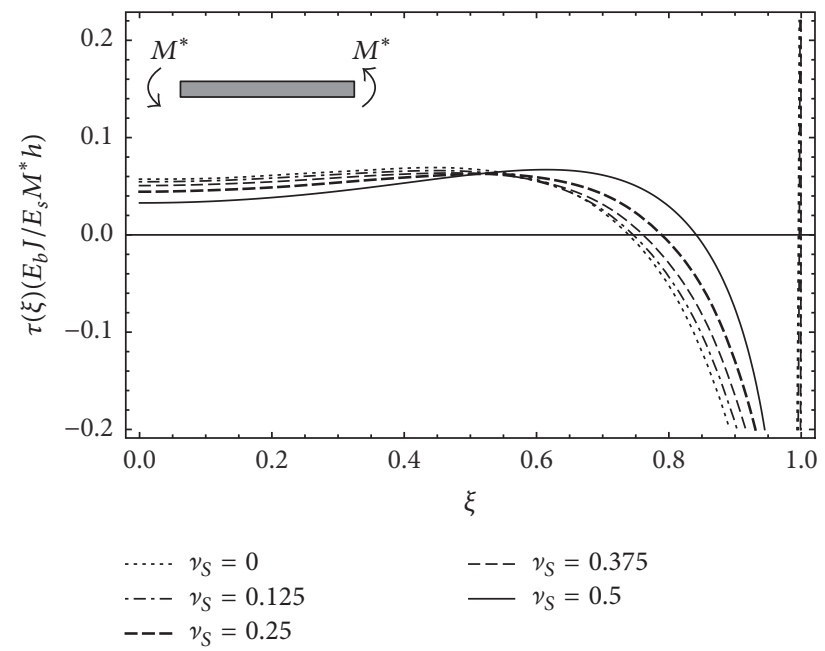

(b) Shear stress for skew-symmetric loading case

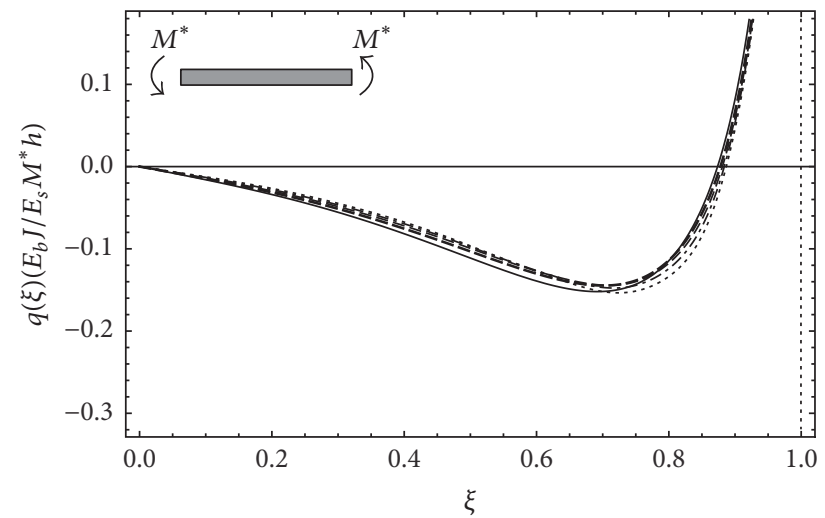

$$
\begin{array}{ll}
\ldots \ldots & v_{S}=0 \\
---v_{S}=0.125 & ---v_{S}=0.375 \\
---v_{S}=0.25 & -v_{S}=0.5
\end{array}
$$

(d) Peeling stress for skew-symmetric loading case

FIgURE 5: Dimensionless mechanical interactions of the beam subjected to couples acting at the edges.

$$
\begin{aligned}
& \int_{-1}^{r}(1+t)^{p}(1-t)^{p} P_{n}^{(p, p)}(t) d t= \begin{cases}\frac{\sqrt{\pi} \Gamma(1+p)}{2 \Gamma((3 / 2)+p)}-r_{2} F_{1}\left(\frac{1}{2},-p, \frac{3}{2}, r^{2}\right), & \text { for } n=0 \\
\frac{1}{2 n} P_{n-1}^{(p+1, p+1)}(r)(1+r)^{p+1}(1-r)^{p+1}, & \text { for } n=1,2, \ldots\end{cases} \\
& \int_{r}^{+1}(1+t)^{p}(1-t)^{p} P_{n}^{(p, p)}(t) d t \\
& \quad= \begin{cases}\frac{\sqrt{\pi} \Gamma(1+p)}{2 \Gamma((3 / 2)+p)}-r_{2} F_{1}\left(\frac{1}{2},-p, \frac{3}{2}, r^{2}\right), & \text { for } n=0 \\
\frac{1}{2 n}\left[\frac{1-(-1)^{n}}{2} P_{n-1}^{(p+1, p+1)}(0)-P_{n-1}^{(p+1, p+1)}(r)(1-r)^{p+1}(1-r)^{p+1}\right], & \text { for } n=1,2, \ldots\end{cases}
\end{aligned}
$$




\section{Competing Interests}

The authors declare that they have no competing interests.

\section{References}

[1] P. Bisegna and R. Luciano, "Bounds on the overall properties of composites with debonded frictionless interfaces," Mechanics of Materials, vol. 28, no. 1-4, pp. 23-32, 1998.

[2] A. M. Tarantino, "Homogeneous equilibrium configurations of a hyperelastic compressible cube under equitriaxial dead-load tractions," Journal of Elasticity. The Physical and Mathematical Science of Solids, vol. 92, no. 3, pp. 227-254, 2008.

[3] N. K. Arutiunian, "Contact problem for a half-plane with elastic reinforcement," Journal of Applied Mathematics and Mechanics, vol. 32, no. 4, pp. 652-665, 1968.

[4] F. Erdogan and G. D. Gupta, "The problem of an elastic stiffener bonded to a Half Plane," Journal of Applied Mechanics, vol. 38, no. 4, pp. 937-941, 1971.

[5] L. Lanzoni, "Analysis of stress singularities in thin coatings bonded to a semi-infinite elastic substrate," International Journal of Solids and Structures, vol. 48, no. 13, pp. 1915-1926, 2011.

[6] N. Tullini, A. Tralli, and L. Lanzoni, "Intefacial shear stress analysis of bar and thin film bonded to 2D elastic substrate using a coupled FE-BIE method," Finite Elements in Analysis and Design, vol. 55, pp. 42-51, 2012.

[7] D. D. Oehlers and R. Seracino, Design of FRP and Steel Plated RC Structures, Elsevier, 2004.

[8] A. Nobili, "Variational approach to beams resting on two-parameter tensionless elastic foundations," Journal of Applied Mechanics, Transactions ASME, vol. 79, no. 2, Article ID 021010, 2012.

[9] A. Nobili, E. Radi, and L. Lanzoni, "A cracked infinite Kirchhoff plate supported by a two-parameter elastic foundation," Journal of the European Ceramic Society, vol. 34, no. 11, pp. 2737-2744, 2014.

[10] V. Salomoni, G. Mazzuocco, C. Pellegrino, and C. E. Majorana, "Threedimensional modeling of bond behaviour between concrete and FRP reinforcement," Engineering Computation, vol. 28, no. 1, pp. 5-29, 2011.

[11] A. Nobili, L. Lanzoni, and A. M. Tarantino, "Experimental investigation and monitoring of a polypropylene-based fiber reinforced concrete road pavement," Construction and Building Materials, vol. 47, pp. 888-895, 2013.

[12] A. Caporale, L. Feo, R. Luciano, and R. Penna, "Numerical collapse load of multi-span masonry arch structures with FRP reinforcement," Composites Part B: Engineering, vol. 54, no. 1, pp. 71-84, 2013.

[13] A. Caporale and R. Luciano, "Limit analysis of masonry arches with finite compressive strength and externally bonded reinforcement," Composites Part B: Engineering, vol. 43, no. 8, pp. 3131-3145, 2012.

[14] G. Dinelli, G. Belz, C. E. Majorana, and B. A. Schrefler, "Experimental investigation on the use of fly ash for lightweight precast structural elements," Materials and Structures, vol. 29, no. 194, pp. 632-638, 1996.

[15] L. B. Freund and S. Suresh, Thin Film Materials. Stress, Defect Formation and Surface Evolution, Cambridge University Press, Cambridge, Uk, 2004.

[16] L. Lanzoni and E. Radi, "Thermally induced deformations in a partially coated elastic layer," International Journal of Solids and Structures, vol. 46, no. 6, pp. 1402-1412, 2009.
[17] V. Guidi, L. Lanzoni, A. Mazzolari, G. Martinelli, and A. Tralli, "Design of a crystalline undulator based on patterning by tensile $\mathrm{Si}_{3} N_{4}$ strips on a Si crystal," Applied Physics Letters, vol. 90, no. 11, Article ID 114107, 2007.

[18] J. F. Luo, Y. J. Liu, and E. J. Berger, "Analysis of two-dimensional thin structures (from micro- To nano-scales) using the boundary element method," Computational Mechanics, vol. 22, no. 5, pp. 404-412, 1998.

[19] A. M. Tarantino, "Nonlinear fracture mechanics for an elastic Bell material," The Quarterly Journal of Mechanics and Applied Mathematics, vol. 50, no. 3, pp. 435-456, 1997.

[20] A. M. Tarantino, "On extreme thinning at the notch-tip of a Neo-Hookean sheet," The Quarterly Journal of Mechanics and Applied Mathematics, vol. 51, no. 2, pp. 179-190, 1998.

[21] M. Ohring, The Materials Science of Thin Films, Academic Press, 1992.

[22] Y. L. Shen, Constrained Deformation of Materials. Devices, Heteroge-Neous Structures and Thermo-Mechanical Modeling, Springer, 2010.

[23] T. W. Shield and K. S. Kim, "Beam theory models for thin film segments cohesively bonded to an elastic half space," International Journal of Solids and Structures, vol. 29, no. 9, pp. 10851103, 1992.

[24] H. Djabella and R. D. Arnell, "Two-dimensional finite-element analysis of elastic stresses in double-layer systems under combined surface normal and tangential loads," Thin Solid Films, vol. 226, no. 1, pp. 65-73, 1993.

[25] A. M. Tarantino, "The singular wedge problem in the nonlinear elastostatic plane stress theory," Quarterly of Applied Mathematics, vol. 57, no. 3, pp. 433-451, 1999.

[26] A. M. Tarantino, "Equilibrium paths of a hyperelastic body under progressive damage," Journal of Elasticity, vol. 114, no. 2, pp. 225-250, 2014.

[27] A. M. Tarantino, "On the finite motions generated by a mode I propagating crack," Journal of Elasticity, vol. 57, no. 2, pp. 85$103,1999$.

[28] A. M. Tarantino, "Crack propagation in finite elastodynamics," Mathematics and Mechanics of Solids, vol. 10, no. 6, pp. 577-601, 2005.

[29] A. Apuzzo, R. Barretta, and R. Luciano, "Some analytical solutions of functionally graded Kirchhoff plates," Composites Part B: Engineering, vol. 68, pp. 266-269, 2015.

[30] R. Barretta, L. Feo, and R. Luciano, "Some closed-form solutions of functionally graded beams undergoing nonuniform torsion," Composite Structures, vol. 123, pp. 132-136, 2015.

[31] R. Barretta, L. Feo, R. Luciano, and F. Marotti de Sciarra, "Variational formulations for functionally graded nonlocal BernoulliEuler nanobeams," Composite Structures, vol. 129, pp. 80-89, 2015.

[32] R. Luciano and J. R. Willis, "Non-local constitutive equations for functionally graded materials," Mechanics of Materials, vol. 36, no. 12, pp. 1195-1206, 2004.

[33] R. Barretta, L. Feo, R. Luciano, and F. Marotti de Sciarra, "A gradient Eringen model for functionally graded nanorods," Composite Structures, vol. 131, pp. 1124-1131, 2015.

[34] K. L. Johnson, Contact Mechanics, Cambridge University Press, London, UK, 1985.

[35] L. Dezi, G. Menditto, and A. M. Tarantino, "Homogeneous structures subjected to repeated structural system changes," ASCE Journal of Engineering Mechanics, vol. 116, no. 8, pp. 17231732, 1990. 
[36] L. Dezi, G. Menditto, and A. M. Tarantino, "Viscoelastic heterogeneous structures with variable structural system," Journal of Engineering Mechanics, vol. 119, no. 2, pp. 238-250, 1993.

[37] L. Dezi and A. Tarantino, "Time dependent analysis of concrete structures with variable structural system," ACI Materials Journal, vol. 88, no. 3, pp. 320-324, 1991.

[38] V. A. Salomoni, C. E. Majorana, B. Pomaro, G. Xotta, and F. Gramegna, "Macroscale and mesoscale analysis of concrete as a multiphase material for biological shields against nuclear radiation," International Journal for Numerical and Analytical Methods in Geomechanics, vol. 38, no. 5, pp. 518-535, 2014.

[39] G. Xotta, G. Mazzucco, V. A. Salomoni, C. E. Majorana, and K. J. Willam, "Composite behavior of concrete materials under high temperatures," International Journal of Solids and Structures, vol. 64, pp. 86-99, 2015.

[40] V. A. Salomoni, C. E. Majorana, G. M. Giannuzzi, and A. Miliozzi, "Thermal-fluid flow within innovative heat storage concrete systems for solar power plants," International Journal of Numerical Methods for Heat and Fluid Flow, vol. 18, no. 7-8, pp. 969-999, 2008.

[41] F. Greco and R. Luciano, "A theoretical and numerical stability analysis for composite micro-structures by using homogenization theory," Composites Part B: Engineering, vol. 42, no. 3, pp. 382-401, 2011.

[42] R. Luciano and J. R. Willis, "Hashin-Shtrikman based FE analysis of the elastic behaviour of finite random composite bodies," International Journal of Fracture, vol. 137, no. 1, pp. 261-273, 2006.

[43] J. C. Mason and D. C. Handscomb, Chebyshev Polynomials, Chapman \& Hall/CRC, Boca Raton, Fla, USA, 2003.

[44] M. Abramowitz and I. A. Stegun, Handbook of Mathematical Functions: With Formulas, Graphs and Mathematical Tables, Dover Publications, New York, NY, USA, 1972.

[45] I. S. Gradshteyn and I. M. Ryzhik, Table of Integrals, Series, and Products, Academic Press, Cambridge, Mass, USA, 7th edition, 2007. 


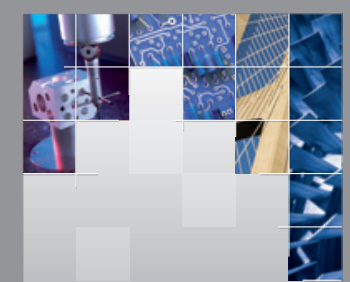

\section{Enfincering}
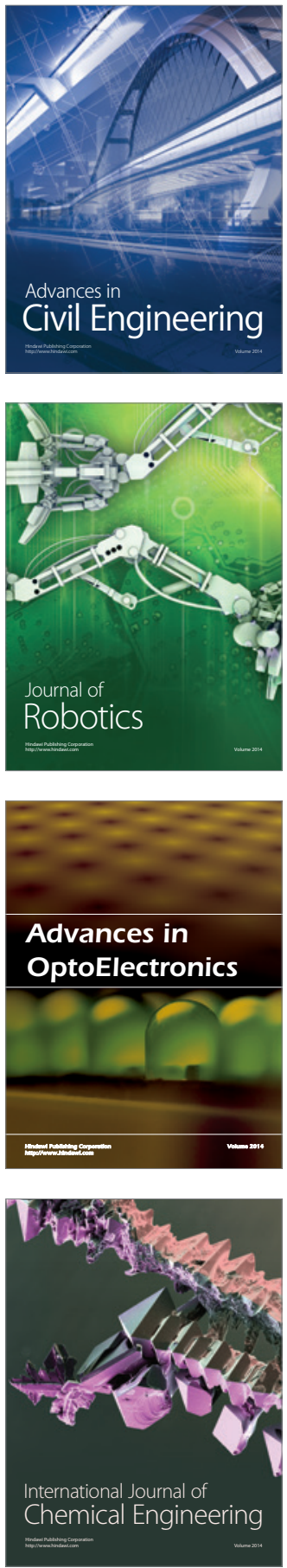

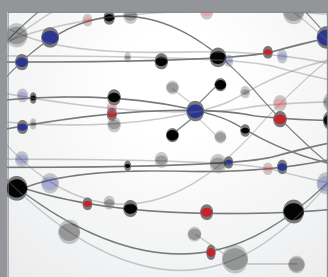

The Scientific World Journal

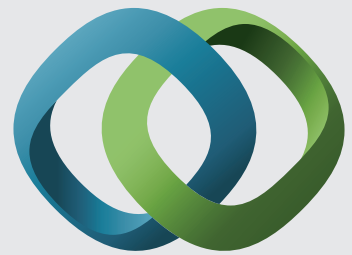

\section{Hindawi}

Submit your manuscripts at

http://www.hindawi.com
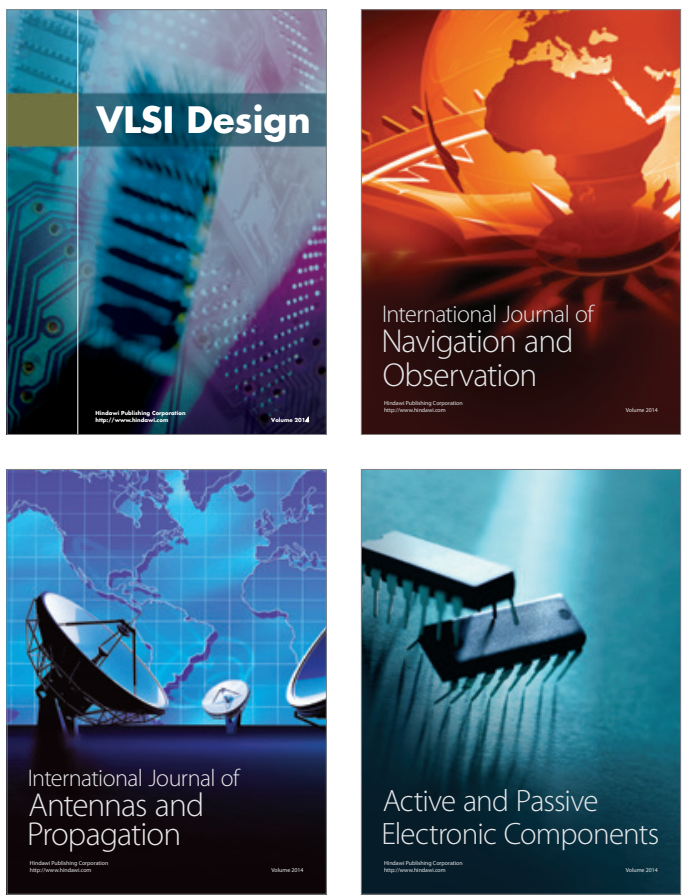
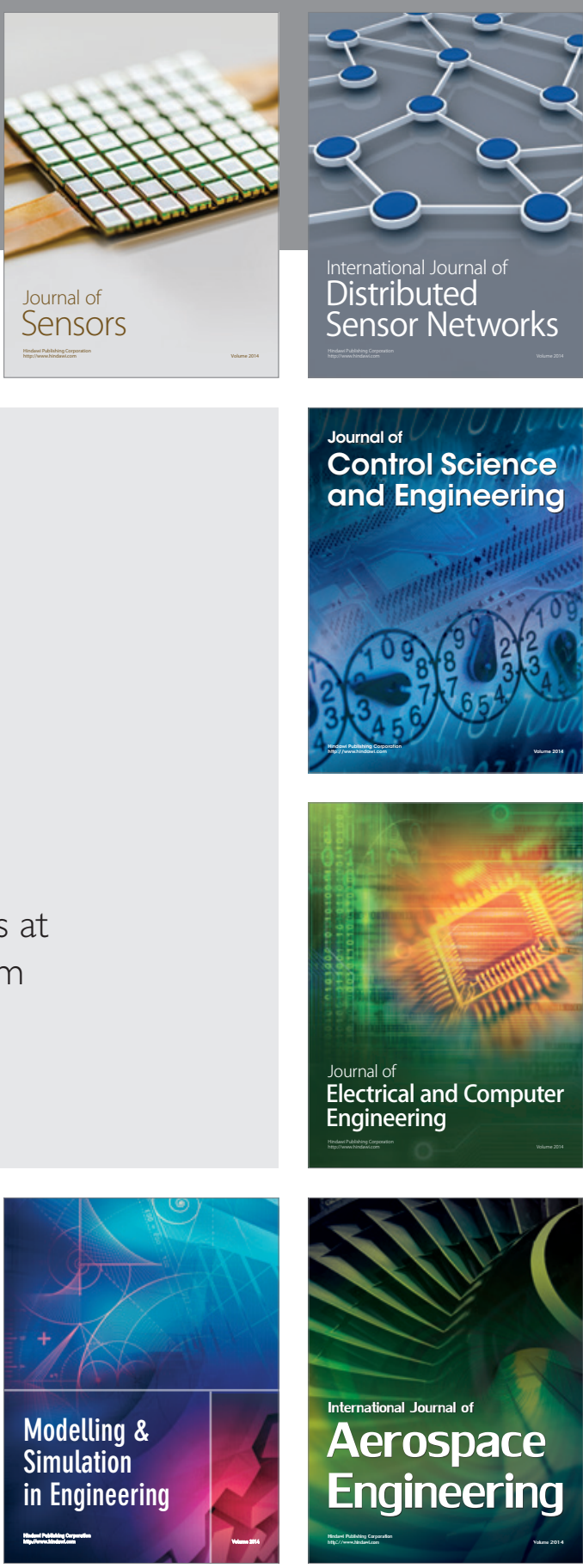

International Journal of

Distributed

Sensor Networks

Journal of

Control Science

and Engineering
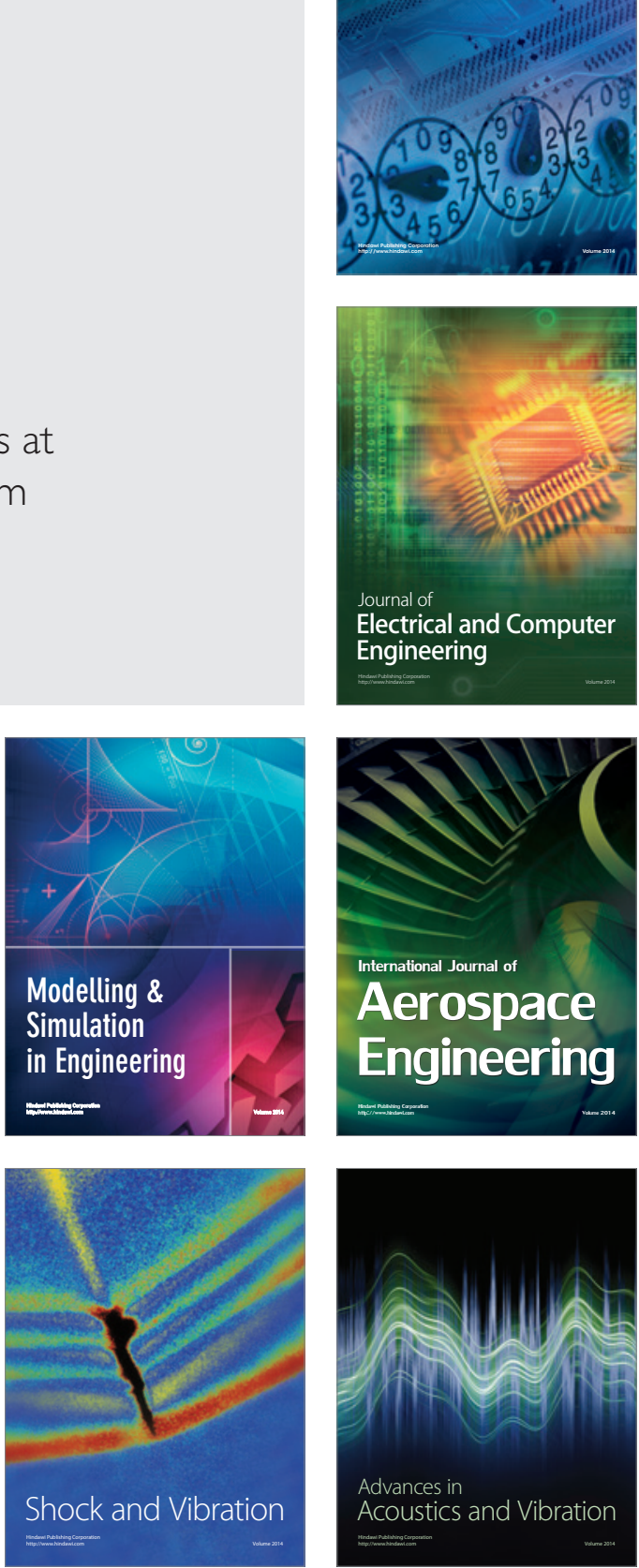